\title{
TLR4/MD-2 is a receptor for extracellular nucleophosmin 1
}

\author{
KOTA NAKATOMI $^{1 *}$, HIKARI UENO $^{1 *}$, YUTO ISHIKAWA ${ }^{1}$, RONNY CHRISTIADI SALIM $^{1}$, \\ YUKI MORI $^{1}$, ISSEY KANEMOTO ${ }^{1}$, SALUNYA TANCHAROEN ${ }^{1,2}$, KIYOSHI KIKUCHI ${ }^{2-4}$, \\ NAOKI MIURA ${ }^{5}$, TAKETO OMORI ${ }^{1}$, EMIKO OKUDA-ASHITAKA ${ }^{1}$, KIYOSHI MATSUMURA ${ }^{1}$, \\ HITOSHI IMAIZUMI ${ }^{6}$, YOSHIHIRO MOTOMIYA ${ }^{7}$, IKURO MARUYAMA ${ }^{1,4}$ and KO-ICHI KAWAHARA ${ }^{1,4}$ \\ ${ }^{1}$ Department of Biomedical Engineering, Osaka Institute of Technology, Osaka 535-8585, Japan; \\ ${ }^{2}$ Department of Pharmacology, Faculty of Dentistry, Mahidol University, Bangkok 10400, Thailand; \\ ${ }^{3}$ Division of Brain Science, Department of Physiology, Kurume University School of Medicine, \\ Kurume, Fukuoka 830-0011; ${ }^{4}$ Department of Systems Biology in Thromboregulation, \\ Kagoshima University Graduate School of Medical and Dental Science, Kagoshima 890-8520; \\ ${ }^{5}$ Veterinary Teaching Hospital, Joint Faculty of Veterinary Medicine, Kagoshima University, \\ Kagoshima 890-8580; ${ }^{6}$ Department of Anesthesiology and Intensive Care Medicine, \\ Tokyo Medical University, School of Medicine, Tokyo 160-0023; \\ ${ }^{7}$ Suiyukai Clinic, Kashihara, Nara 634-0007, Japan
}

Received February 12, 2020; Accepted November 13, 2020

DOI: $10.3892 /$ br.2020.1397

\begin{abstract}
Nucleophosmin 1 (NPM1) primarily localizes to the nucleus and is passively released into the extracellular milieu by necrotic or damaged cells, or is secreted by monocytes and macrophages. Extracellular NPM1 acts as a potent inflammatory stimulator by promoting cytokine production [e.g., tumor necrosis factor- $\alpha$ (TNF- $\alpha)$ ], which suggests that NPM1 acts as a damage-associated molecular pattern. However, the receptor of NPM1 is unknown. Evidence indicates that DAMPs, which include high mobility group box 1 and histones, may bind Toll-like receptors (TLRs). In the present study, it was shown that NPM1 signaling was mediated via the TLR4 pathway, which suggests that TLR4 is an NPM1 receptor. TLR4 binds myeloid differentiation protein-2 (MD-2), which is essential for intracellular signaling. Furthermore, the TLR4 antagonist, LPS-Rhodobacter sphaeroides (an MD-2 antagonist) and TAK-242 (a TLR4 signaling inhibitor) significantly inhibited NPM1-induced TNF- $\alpha$ production by differentiated THP-1 cells as well as reducing ERK1/2 activation. Far-western blot analysis revealed that NPM1 directly bound MD-2. Thus, the results of the present study provide compelling evidence
\end{abstract}

Correspondence to: Professor Ko-ichi Kawahara, Department of Biomedical Engineering, Osaka Institute of Technology, 5-16-1 Omiya, Asahi-ku, Osaka 535-8585, Japan

E-mail: koichi.kawahara@oit.ac.jp

*Contributed equally

Key words: damage-associated molecular patterns, myeloid differentiation protein-2, nucleophosmin 1 , Toll-like receptor 4 , sepsis that TLR4 binds NPM1, and it is hypothesized that inhibiting NPM1 activity may serve as a novel strategy for treating TLR4-related diseases.

\section{Introduction}

Damage-associated molecular patterns (DAMPs) are associated with inflammatory diseases such as sepsis, rheumatoid arthritis (RA), atherosclerosis, cerebral infarction and periodontitis (1-4). In general, in living cells, the presence of DAMPs in the intracellular space is physiologically normal and are not harmful, as they contribute to processes associated with cell maintenance, such as cell cycle progression, DNA construction and gene expression (5-7). However, proteins released by damaged or necrotic cells, including DAMPs, can be dangerously proinflammatory, causing cytotoxicity to living cells (5-7). DAMPs include cellular molecules, such as the nuclear proteins, high mobility group box 1 (HMGB1), histones $\mathrm{H} 3$ and H4, and nucleophosmin 1 (NPM1) (1-6). The involvement of HMGB1 was first demonstrated in a patient with sepsis (7). Histones H3 and H4 induce platelet activation and endothelial cell death (6). Both NPM1 and HMGB1 induce the production of inflammatory cytokines, such as tumor necrosis factor (TNF)- $\alpha$, interleukin (IL)-6 and IL-8. DAMPs negatively influence the prognosis of inflammatory diseases (1-7). As their activity may have fatal consequences for patients with inflammatory diseases $(6,7)$, regulating DAMPs is essential.

In our previous study, it was shown that the ubiquitously expressed nucleolar phosphoprotein, NPM1 (also known as B23, numatrin and NO38) (8), was a novel DAMP (5). Intracellular NPM1 regulates ribosome biogenesis, the response to genotoxic stress and the inhibition of hypoxia-induced apoptosis (8), thus NPM1 also contributes to cell maintenance. NPM1, which is more abundant in tumor 
cells than in normal resting cells, shuttles between the nucleus and cytoplasm during the cell cycle $(8,9)$. Thus, NPM1 is an essential molecule in living cells.

NPM1 has been shown to be released from damaged or activated murine macrophage-like RAW264.7 cells (5). Extracellular NPM1 acts as an inflammatory cytokine by inducing the production of the inflammatory cytokine, TNF- $\alpha$, via ERK-1/2 activation in RAW264.7 cells, but not via the kinases, c- JNK and p38 MAPK (5). Furthermore, in the sepsis model, cecal ligation and puncture (CLP), NPM1 was detected in serum derived from model rats, but not in serum from control rats (5). Thus, in the extracellular space, NPM1 may act as a cytokine as well as a novel DAMP. However, the NPM1 receptor on the cell membrane has not yet been identified.

The ligands of 9 out of 10 human homologs of Toll-like receptors (TLRs) have been identified as DAMPs (10-12). TLR4 was first identified as a DAMP receptor, and is the most extensively studied receptor amongst the TLR family. TLR4 associated with myeloid differentiation protein (MD)-2 (TLR4/MD-2) is a receptor for lipopolysaccharide (LPS), which is a component of the outer membrane of Gram-negative bacteria (13). The effects of LPS are mediated via TLR4/MD-2 expressed by endothelial cells as well as immune cells (macrophages and dendritic cells). Apart from LPS, TLR4 is activated by endogenous molecules, including DAMPs, such as HMGB1, histone H3 and histone $\mathrm{H} 4$ (10-12). In the TLR4/MD-2 signal transduction pathway, TLR4/MD-2 dimerizes with another TLR4/MD-2 and recruits specific intracellular adaptor molecules to promote the activation of downstream signaling pathways. These pathways include the myeloid differentiation primary response gene 88 (MyD88)-dependent pathway and the MyD88-independent pathway, which includes Toll/IL-1 receptor (TIR)-domain containing adaptor-inducing interferon- $\beta$ (TRIF). Both pathways activate nuclear factor- $\mathrm{\kappa B}(\mathrm{NF}-\mathrm{\kappa B})$ signaling, but only the TRIF pathway stimulates signaling by interferon regulatory factor 3 . These pathways may induce the production of inflammatory cytokines, such as TNF- $\alpha$, IL-6 and IL-8 (13). Thus, identifying TLR4/MD-2 ligands may be a novel strategy for treating inflammatory diseases, such as sepsis, cancer and RA. Indeed, molecules targeting TLR4/MD-2 have been developed; for example, LPS-RS as an MD-2 antagonist and TAK-242 as an intracellular signaling inhibitor of TLR4 (14-17).

In the present study, whether TLR4/MD-2 was the receptor for extracellular NPM1 was assessed. To this end, a reporter gene assay with TLR4/MD-2-expressing cells and control 293 cells was used, and TNF- $\alpha$ production was measured using phorbol 12-myristate 13-acetate (PMA)-differentiated THP-1 cells. Furthermore, using LPS-RS and TAK-242, whether TNF- $\alpha$ production upon NPM1 stimulation was mediated via TLR4/MD-2, and whether stimulation activated the TLR4/MD-2/ERK1/2 signaling pathway, was investigated. Additionally, far-western blotting was used to examine direct binding of NPM1 to MD-2. The results showed that NPM1 binds to MD-2 to induce TNF- $\alpha$ production.

\section{Materials and methods}

Reagents. Bacterial expression vectors (pGEX6p-1) encoding the fusion proteins, GST-NPM1 or GST-MD-2, were purchased from GenScript. These proteins were affinity-purified using
glutathione-Sepharose beads and cleaved by Turbo3C protease to remove the GST from the indicated protein, according to the manufacturer's protocol (Nacalai Tesque, Inc.). Unless indicated otherwise, PMA and other reagents were purchased from Sigma-Aldrich (Merck KGaA). LPS-Rhodobacter sphaeroides (RS) (TLR4 antagonist) and TAK-242 (a TLR4 signaling inhibitor) were purchased from InvivoGen.

Cell culture. THP-1 cells were obtained from the American Type Culture Collection and maintained in RPMI-1640 medium (Nacalai Tesque, Inc.) supplemented with 10\% FBS and $2 \mathrm{mM}$ glutamine. A total of $4 \times 10^{5}$ cells $/ \mathrm{ml}$ were treated with $10 \mathrm{nM}$ PMA for $16 \mathrm{~h}$ (18), and then treated with recombinant NPM1 (rNPM1), as indicated in the figures.

Reporter gene assay. To assess whether NPM1 activates the TLR4/MD-2 pathway, human TLR4/NF-kB/SEAP reporter 293 cells (Blue hTLR4 cells), in which the expression of the SEAP reporter gene is controlled by an $I L-12$ p 40 minimal promoter fused to five NF- $\mathrm{KB}$ and AP-1-binding sites (InvivoGen) were used. Treating these cells with a TLR4 ligand activates NF- $\mathrm{KB}$ and AP-1, which induces the production of SEAP. In parallel, mock plasmid-transfected 293 cells (Null) was used as a TLR4-negative control. Briefly, $50 \mathrm{ng} / \mathrm{ml} \mathrm{NPM1}$ (ATGen, Ltd.) was added to cultures of Blue hTLR4 cells or Null cells for $16 \mathrm{~h}$. TLR4 signaling was evaluated by measuring SEAP activity at an optical density of $650 \mathrm{~nm}$ in Quanti-Blue reagent (InvivoGen). Experiments were repeated three times.

Treatment with purified NPM1 or heat-inactivated purified NPM1. THP-1 cells were incubated with $50 \mathrm{nM}$ PMA for $24 \mathrm{~h}$ and then washed with Opti-MEM (Thermo Fisher Scientific, Inc.). The cells were then incubated with native NPM1 or heat-inactivated NPM $1\left(100^{\circ} \mathrm{C}\right.$ for $\left.5 \mathrm{~min}\right)$ at a final concentration of $5 \mathrm{nM}$ for $16 \mathrm{~h}$. Experiments were repeated nine times.

Effects of rNPM1 in the presence or absence of LPS-RS or $T A K-242$. THP-1 cells were incubated with $50 \mathrm{nM}$ PMA for $24 \mathrm{~h}$ and then washed with Opti-MEM (Thermo Fisher Scientific, Inc.). The cells were then incubated with or without the TLR4 inhibitor, LPS-RS or TAK-242. NPM1 was subsequently added to the cells (final concentration, $5 \mathrm{nM}$ ) for $16 \mathrm{~h}$. Experiments were repeated nine times.

Detection of ERK1/2 phosphorylation. THP-1 cells were treated with PMA for $24 \mathrm{~h}$, washed and then incubated with $100 \mathrm{ng} / \mathrm{ml}$ LPS-RS or $100 \mathrm{nM}$ TAK-242 for $2 \mathrm{~h}$, based on a previous study (5). Subsequently, NPM1 was added to the cells (final concentration, $5 \mathrm{nM}$ ) for $30 \mathrm{~min}$. Protein concentrations was determined using a Bradford assay (Bio Rad Laboratories, Inc.). The treated cells were then washed with cold PBS, and $200 \mu 1$ cell lysis buffer containing $62.5 \mathrm{mM}$ Tris- $\mathrm{HCl}$ (pH 6.8), 2\% SDS, $10 \%$ glycerol and $0.002 \%$ bromophenol blue were added. A total of $2 \mu \mathrm{g}$ of the above proteins in the resultant lysates $(200 \mu \mathrm{l})$ were loaded on a SDS-gel, resolved using SDS-PAGE and transferred to a nitrocellulose membrane (GE Healthcare). After blocking with Block One (Nacalai Tesque, Inc.) for $1 \mathrm{~h}$ at room temperature, the membrane was incubated with anti-phospho (p)-ERK1/2 (1:1,000; cat. no. 4370S) or anti-total (t)-ERK1/2 antibodies (Cell Signaling Technology, Inc.; 1:1,000; cat. no. 9102S) at 
$4^{\circ} \mathrm{C}$ for $16 \mathrm{~h}$. Subsequently, the membranes were incubated with horse radish peroxidase (HRP)-conjugated anti-rabbit antibodies (Santa Cruz Biotechnology Inc.; cat. no. sc-2357) for $1 \mathrm{~h}$ at room temperature. After washing, signals were detected using ImmunoStar Zeta reagent (FUJIFILM) and measured using Image J version 1.52a (National Institutes of Health).

Determination of cytokine production. ELISA kits (BioLegend, Inc.; cat. no. 430201) were used to quantify the TNF- $\alpha$ concentration in cell-free supernatants, according to the manufacturer's protocol. Absorbance at $450 \mathrm{~nm}$ was measured using a microplate reader. The sensitivity of the commercial ELISA kit was $15.6 \mathrm{pg} / \mathrm{ml}$ for TNF- $\alpha$.

Coomassie Brilliant Blue (CBB) staining. GST-MD-2 induced using isopropyl- $\beta$-D-thiogalactopyranoside (Nacalai Tesque, Inc.) was concentrated using Centrifugal Filter Units (10,000 Da cut off; EMD Millipore). Concentrated GST-MD-2 was mixed with an equal volume of lysis buffer and boiled for $5 \mathrm{~min}$. These samples $(40 \mu \mathrm{l})$ were loaded onto a $12 \%$ SDS-gel, resolved using SDS-PAGE and subsequently, the gels were stained with CBB (Nacalai Tesque, Inc.), as described previously (5).

Interaction between NPM1 and MD-2. The GST fusion proteins were expressed and purified using glutathione-Sepharose beads (19). NPM1 was cleaved from GST bound to the column using Turbo3C Protease (Nacalai Tesque, Inc.). NPM1 was purified using LPS-free $0.9 \% \mathrm{NaCl}$. The interaction between NPM1 and GST-MD-2 was measured as previously described (20). NPM1 (500 ng) was separated using a 15\% SDS-gel and SDS-PAGE, and then transferred to a nitrocellulose membrane. After blocking with Block One, the membrane was incubated with $6 \mu \mathrm{g} / \mathrm{ml} \mathrm{GST-MD}-2$ or GST at $4^{\circ} \mathrm{C}$ for $16 \mathrm{~h}$. Subsequently, the membrane was incubated with an anti-GST antibody (Medical \& Biological Laboratories Co., Ltd.; 1:1,000; cat. no. PM013-7) for $1 \mathrm{~h}$ at room temperature. After washing, the membrane was incubated with HRP-conjugated anti-rabbit-IgG antibodies (Santa Cruz Biotechnology, Inc.; 1:5,000; cat. no. sc-2357) for $1 \mathrm{~h}$ at room temperature and the immune complexes were detected using ImmunoStar Zeta reagent (FUJIFILM).

Statistical analysis. All statistical analysis was performed using GraphPad version 8 (GraphPad Software, Inc.). Data are presented as the mean \pm the standard error of the mean. Differences between groups were evaluated using a one-way ANOVA with a post-hoc Tukey's test. $\mathrm{P}<0.05$ was considered to indicate a statistically significant difference.

\section{Results}

TLR4/MD-2 mediates the effects of extracellular NPM1. To determine the effects of extracellular NPM1 on 293-TLR4-Blue cells and Null cells, culture supernatants were assayed for SEAP activity. NPM1 significantly increased the SEAP activity in 293-TLR4-Blue cells (13-fold increase; $\mathrm{P}<0.0001$ ), but not in the Null cells (Fig. 1).

TAK-242 and LPS-RS inhibits NPM1-induced TNF- $\alpha$ production and ERK1/2 activation. Purified NPM1 was detected as a single band in CBB-stained gels (Fig. 2A, left CBB),

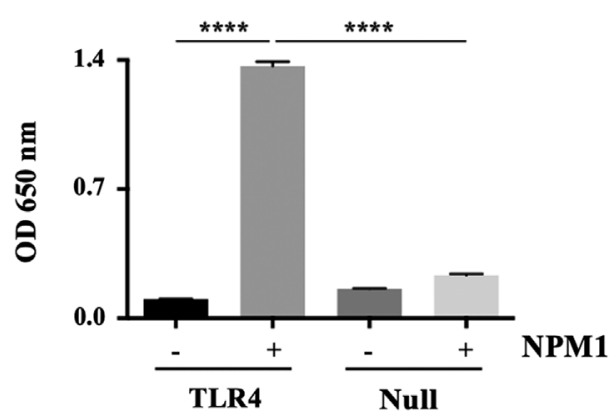

Figure 1. TLR4/MD-2 mediates the effects of NPM1. 293 cells transfected with a human TLR4 expression vector or a mock vector were incubated with or without NPM1. SEAP activity in culture supernatants were determined using Quanti-Blue reagent. Data are presented as the mean \pm standard error of the mean of three independent experiments. ${ }^{* * * * *} \mathrm{P}<0.0001$. Null, mock vector; NPM1, nucleophosmin 1; TLR, toll-like receptor; MD-2, myeloid differentiation protein-2; OD, optical density.
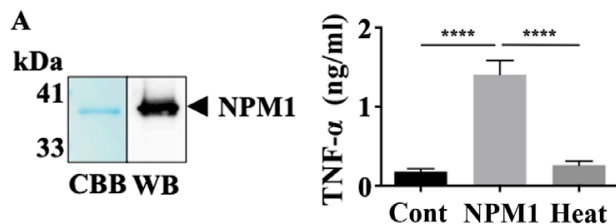

B
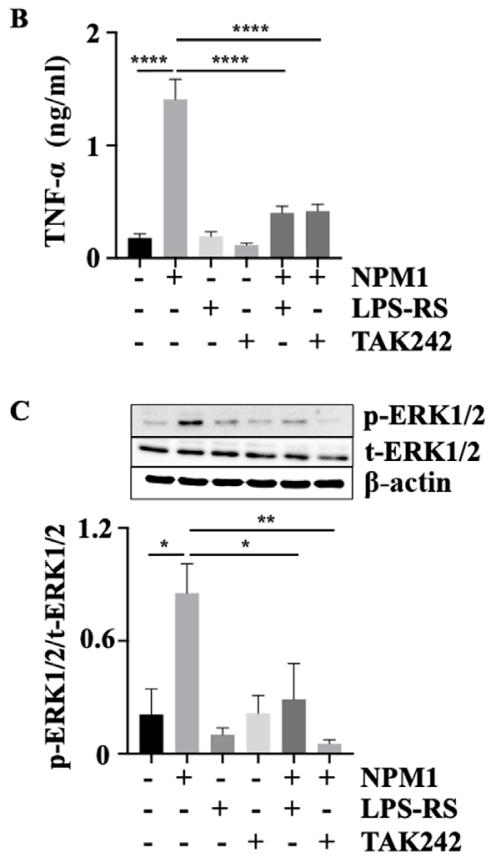

Figure 2. TAK-242 and LPS-RS inhibits NPM1 signaling. (A) SDS-PAGE and western blotting analysis of purified recombinant NPM1 (left). PMA-differentiated THP-1 cells were incubated with intact NPM1 or heat-inactivated NPM1. TNF- $\alpha$ levels in the culture supernatants were determined (right). $n=9$ per group. (B) TNF- $\alpha$ levels in culture supernatants of PMA-differentiated THP-1 cells incubated with or without the MD-2 antagonist LPS-RS or the TLR4 signaling inhibitor TAK-242, followed by addition of NPM1. $n=9$ per group. (C) ERK1/2 phosphorylation levels in PMA-differentiated THP-1 cells incubated with or without LPS-RS or TAK-242 followed by addition of NPM1. $n=4$ per group. The upper panel shows a representative western blotting of activated ERK1/2 (p-ERK1/2) and $\mathrm{t}$-ERK1/2 following the indicated treatments with or without NPM1, LPS-RS and/or TAK-242. $\beta$-actin was used as the loading control. The lower panel shows quantitative analysis of the p-ERK1/2/t-ERK1/2 expression ratio. Data are presented as the mean \pm standard error of the mean of three independent experiments. ${ }^{*} \mathrm{P}<0.05,{ }^{* *} \mathrm{P}<0.01,{ }^{* * * * *} \mathrm{P}<0.0001$. NPM1, nucleophosmin 1 ; Heat, heat-inactivated NPM1; PMA, phorbol 12-myristate 13-acetate; TLR, toll-like receptor; MD-2, myeloid differentiation protein-2; p- phospho; $\mathrm{t}$, total; CBB, Coomassie Brilliant Blue; WB, western blotting. 
A

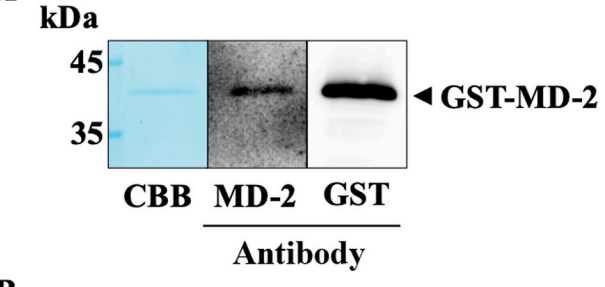

B

kDa CBB

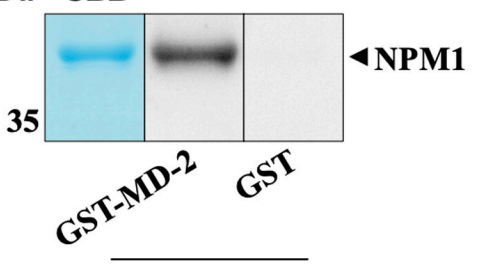

Probe

Figure 3. NPM1 binds MD-2. (A) GST-MD-2 was subjected to $12 \%$ SDS-PAGE. Gels were stained with CBB (left) and blots were probed with anti-MD-2 (middle) or anti-GST (right) antibodies. (B) NPM1 was subjected to SDS-PAGE and western blotting. The membranes were incubated with a GST-MD-2 or GST as the probe. Subsequently, it was incubated with anti-GST antibodies, followed by incubation with an horseradish peroxidase-conjugated anti-rabbit $\operatorname{IgG}$ antibody. $n=3$ per group. NPM1, nucleophosmin 1; MD-2, myeloid differentiation protein-2; CBB, Coomassie Brilliant Blue; WB, western blotting.

corresponding to the specific band detected by the anti-NPM1 antibody (Fig. 2A, left western blotting). Using the purified NPM1, whether NPM1 specifically induced TNF- $\alpha$ production in PMA-differentiated THP-1 cells was next investigated. As shown in Fig. 2A (right), the native NPM1 significantly induced TNF- $\alpha$ production, whereas the heated NPM1 did not.

Subsequently, whether LPS-RS and TAK-242 inhibited TNF- $\alpha$ production stimulated by $5 \mathrm{nM}$ NPM1 in PMA-differentiated THP-1 cells was assessed. NPM1 significantly induced TNF- $\alpha$ production compared with the control (8-fold-increase; $\mathrm{P}<0.0001)$, but not when the cells were pretreated with LPS-RS or TAK-242 (Fig. 2B). In our previous study, it was shown that TNF- $\alpha$ production upon NPM1 stimulation was mediated via ERK1/2 activation, but not via other kinases, such as JNK and p38 MAPK (5). Thus, whether these two inhibitors prevented ERK1/2 activation by NPM1 stimulation was assessed. As shown by the p-ERK1/2 to t-ERK1/2 ratio in Fig. 2C, pretreatment with LPS-RS or TAK-242 significantly suppressed the levels of NPM1-induced p-ERK1/2 (NPM1 vs. NPM1+LPS-RS, 3-fold-decrease, $\mathrm{P}<0.05$; NPM1 vs. NPM1+TAK242, 16-fold-decrease, $\mathrm{P}<0.01$ ), which suggests that NPM1 signaling through TLR4/MD-2 activated the ERK1/2 signaling pathway.

NPM1 binds MD-2. Next, far-western blotting was used to determine whether NPM1 directly binds MD-2. GST-MD-2 was purified and detected as a single band in CBB-stained gels (Fig. 3A, left), corresponding to the specific band detected by the anti-MD-2 antibody (Fig. 3A, middle) and the anti-GST antibody (Fig. 3A, right) in western blotting experiments. NPM1 was subjected to SDS-PAGE, and then transferred to a nitrocellulose membrane. The membrane was incubated with GST-MD-2 or GST for $16 \mathrm{~h}$ at $4^{\circ} \mathrm{C}$, and then with the anti-GST antibody for $1 \mathrm{~h}$ at room temperature. GST-MD-2 bound NPM1 on the membrane at the respective position in the CBB-stained gels, but GST was not detectable (Fig. 3B). These results showed that NPM1 bound GST-MD-2, but not GST, which suggests that NPM1 signaling is mediated via TLR4/MD-2.

\section{Discussion}

In the present study, PMA-differentiated THP-1 cells were used to show that TLR4/MD-2 serves as a receptor for the extracellular DAMP, NPM1. The interaction between NPM1 and TLR4/MD-2 was detected using a SEAP reporter gene assay. The MD-2 antagonist, LPS-RS, and the TLR4 signaling inhibitor, TAK-242, significantly inhibited NPM1-induced TNF- $\alpha$ production and ERK1/2 activation. Furthermore, far-western blotting analysis revealed that NPM1 directly bound MD-2, which further indicates that NPM1 signaling was mediated through the activation of TLR4/MD-2 signaling.

Host cells infected with Gram-negative bacteria are exposed to LPS, which strongly induces the activation of TLR4/MD-2 signaling in specific target cells such as macrophages and monocytes $(21,22)$. TLR4/MD-2 signaling recruits MyD88, which leads to activation of the MAPKs and $\mathrm{NF}-\kappa \mathrm{B}$ signaling pathways. Furthermore, TLR4 ligands include cellular proteins that act as DAMPs, such as histones H3 and H4 as well as HMGB1 (10-12), which suggests that TLR4 signaling may serve as a therapeutic target for treating inflammatory diseases, such as sepsis and RA. However, the full spectrum of TLR4 ligands remains to be identified.

In our previous study, it was shown that NPM1 may act as a DAMP (5). NPM1 induces the production of the proinflammatory cytokines, TNF- $\alpha$, IL-6 and IL-8, which in turn activates MAPKs, such as ERK1/2, p38 MAPK and JNK $1 / 2$. ERK1/2 activation contributes to the induction of TNF- $\alpha$ (5). Moreover, NPM1 is detected in the serum in a rat model of sepsis (CLP), which suggests that NPM1 may act as a DAMP (5). Additionally, it was shown that NPM1, but not heat-inactivated NPM1, induced TNF- $\alpha$ production, which suggests that TNF- $\alpha$ production was induced by NPM1, but not by other bacterial components.

In the present study, NPM1 signaling, indicated by TNF- $\alpha$ production, was mediated via the TLR4/MD-2 signaling pathway. Firstly, TNF- $\alpha$ production stimulated by NPM1 was significantly inhibited by the MD-2 antagonist, LPS-RS. LPS-RS, which is a penta-acylated LPS, was identified as a potent antagonist of LPS-induced toxicity (14). LPS-RS is a competitive inhibitor of LPS via direct binding to MD-2 (14-17). More recently, it has been shown that the dimerization of TLR4/MD-2 is essential for activation of TLR4 signaling. Indeed, the dimerization ratio of TLR4/MD-2 increased by $48 \%$ in LPS-stimulated cells (23). However, treatment with LPS-RS completely inhibited this dimerization (23), thereby inhibiting TLR4/MD-2 signaling. Additionally, in the present study, TNF- $\alpha$ production by NPM1 stimulation was significantly inhibited by TAK-242. The cyclohexane derivative, TAK-242, selectively inhibits TLR4 signaling (15) through binding to the TIR domain of TLR4 via Cys747 (16). Binding of TAK-242 to the TLR4 intracellular domain disrupts the interaction of TLR4 with its adaptor 
molecules TIR domain-containing adaptor protein and TIR domain-containing adaptor including interferon- $\beta$-related adaptor molecule (17). Furthermore, far-western blotting demonstrated that NPM1 directly bound MD-2, and thus NPM1 may induce TLR4/MD-2 dimerization. Finally, NPM1 has been shown to activate MAPKs, including ERK1/2, JNK and p38 MAPK (5). However, TNF- $\alpha$ production was only mediated via ERK1/2, but not the other kinases (5). The present study examined whether TAK-242 and LPS-RS inhibited NPM1-induced ERK1/2 activation. It was shown that both inhibitors significantly inhibited NPM1-stimulated ERK1/2 activation. Consistently, ERK1/2 was not activated in LPS-treated THP-1 cells transfected with TLR4 small interfering RNA (24). Furthermore, TNF- $\alpha$ production has been shown to be mediated via ERK1/2 activation by TLR4/MD-2 in vitro and in vivo $(25,26)$. Taken together, the results indicate that TNF- $\alpha$ production by NPM1 stimulation may be mediated via the TLR4/MD-2/ERK1/2 signal transduction pathway.

NPM1 belongs to a chaperone family, which comprises multiple major functional members (NPM1, NPM2 and NPM3) in the intracellular space (27). Residues in the N-terminal domain (Met9 to Asp122) are highly conserved and essential for its oligomerization and interactions with other proteins (8). Furthermore, NPM1, which exists as a pentamer via the $\mathrm{N}$-terminal domain, interacts with other pentamers, and two NPM1 pentamers interact in a head-to-head manner to form a decamer in the nucleus. The decamer is modulated by numerous post-translational modifications, especially phosphorylation (28). In the extracellular space, the pentamer structure of NPM1 may be essential because heat-inactivated NPM1 did not induce TNF- $\alpha$ production. However, the NPM1 decamer did not affect the interaction between NPM1 and MD-2 in the far-western blotting. These findings suggest that the interaction between NPM1 and TLR4 may be mediated via the decamer form of NPM1, but not the interaction between NPM1 and MD-2. This discrepancy will be investigated in future studies.

To the best of our knowledge, the present study is the first report to demonstrate that the NPM1 receptor is TLR4/MD-2. NPM1 released from damaged or activated cells potentially exacerbate the effects of inflammatory diseases. Furthermore, NPM1 may contribute to the accumulation of inflammatory cells in TLR4-related inflammatory diseases, such as arterial thrombosis, RA, atherosclerosis and type II diabetes mellitus as well as sepsis (29-32). These findings may contribute to efforts to develop novel and more effective treatments for inflammatory diseases by targeting TLR4 and components of its signaling pathway.

\section{Acknowledgements}

We would like to thank Miss Kazumi Tamura, Mrs. Kazumi Sugiyama, and Mrs. Misako Tsujimoto for their excellent technical assistance.

\section{Funding}

This study was supported by a Grant-in-aid from KAKENHI (grant no. 18H02906).

\section{Availability of data and materials}

The datasets used and/or analyzed during the current study are available from the corresponding author on reasonable request.

\section{Authors' contributions}

KN, HU, ST, KK, NM, TO, EOA, KM, YoM, HI, IM, and KIK conceived and designed the study, and were responsible for the interpretation of the results and writing the manuscript. $\mathrm{KN}$, HU, YI, RCS, YuM, IK, and ST analyzed and interpreted the data and helped prepare the manuscript. All authors read and approved the final manuscript.

\section{Ethics approval and consent to participate}

Not applicable.

\section{Patient consent for publication}

Not applicable.

\section{Competing interests}

The authors declare that they have no competing interests.

\section{References}

1. Ito T, Totoki T, Yokoyama Y, Yasuda T, Furubeppu H, Yamada S, Maruyama I and Kakihana Y: Serum histone H3 levels and platelet counts are potential markers for coagulopathy with high risk of death in septic patients: A single-center observational study. J Intensive Care 7: 63, 2019.

2. Roh JS and Sohn DH: Damage-associated molecular patterns in inflammatory diseases. Immune Netw 18: e27, 2018.

3. Kikuchi K, Kawahara K, Tancharoen S, Matsuda F, Morimoto Y, Ito T, Biswas KK, Takenouchi K, Miura N, Oyama Y, et al: The free radical scavenger edaravone rescues rats from cerebral infarction by attenuating the release of high-mobility group box-1 in neuronal cells. J Pharmacol Exp Ther 329: 865-874, 2009.

4. Morimoto Y, Kawahara KI, Tancharoen S, Kikuchi K, Matsuyama T, Hashiguchi T, Izumi Y and Maruyama I: Tumor necrosis factor-alpha stimulates gingival epithelial cells to release high mobility-group box 1. J Periodontal Res 43: 76-83, 2008.

5. Nawa Y, Kawahara K, Tancharoen S, Meng X, Sameshima H, Ito T, Masuda Y, Imaizumi H, Hashiguchi T and Maruyama I: Nucleophosmin may act as an alarmin: Implications for severe sepsis. J Leukoc Biol 86: 645-653, 2009.

6. Xu J, Zhang X, Pelayo R, Monestier M, Ammollo CT, Semeraro F, Taylor FB, Esmon NL, Lupu F and Esmon CT: Extracellular histones are major mediators of death in sepsis. Nat Med 15: 1318-1321, 2009.

7. Wang H, Bloom O, Zhang M, Vishnubhakat JM, Ombrellino M, Che J, Frazier A, Yang H, Ivanova S, Borovikova L, et al: HMG-1 as a late mediator of endotoxin lethality in mice. Science 285 : 248-251, 1999.

8. Box JK, Paquet N, Adams MN, Boucher D, Bolderson E, O'Byrne KJ and Richard DJ: Nucleophosmin: From structure and function to disease development. BMC Mol Biol 17: 19, 2016.

9. Borer RA, Lehner CF, Eppenberger HM and Nigg EA: Major nucleolar proteins shuttle between nucleus and cytoplasm. Cell 56: 379-390, 1989.

10. Yang H, Wang H, Ju Z, Ragab AA, Lundbäck P, Long W, Valdes-Ferrer SI, He M, Pribis JP, Li J, et al: MD-2 is required for disulfide HMGB1-dependent TLR4 signaling. J Exp Med 212: $5-14,2015$.

11. Xu J, Zhang X, Monestier M, Esmon NL and Esmon CT: Extracellular histones are mediators of death through TLR2 and TLR4 in mouse fatal liver injury. J Immunol 187: 2626-2631, 2011. 
12. He M, Bianchi ME, Coleman TR, Tracey KJ and Al-Abed Y: Exploring the biological functional mechanism of the HMGB1/TLR4/MD-2 complex by surface plasmon resonance. Mol Med 24: 21, 2018.

13. Kuzmich NN, Sivak KV, Chubarev VN, Porozov YB, Savateeva-Lyubimova TN and Peri F: TLR4 signaling pathway modulators as potential therapeutics in inflammation and sepsis. Vaccines (Basel) 5: 34, 2017.

14. Coats SR, Pham TT, Bainbridge BW, Reife RA and Darveau RP: MD-2 mediates the ability of tetra-acylated and penta-acylated lipopolysaccharides to antagonize Escherichia coli lipopolysaccharide at the TLR4 signaling complex. J Immunol 175: 4490-4498, 2005.

15. Takashima K, Matsunaga N, Yoshimatsu M, Hazeki K, Kaisho T, Uekata M, Hazeki O, Akira S, Iizawa Y and Ii M: Analysis of binding site for the novel small-molecule TLR4 signal transduction inhibitor TAK-242 and its therapeutic effect on mouse sepsis model. Br J Pharmacol 157: 1250-1262, 2009.

16. Matsunaga N, Tsuchimori N, Matsumoto T and Ii M: TAK-242 (resatorvid), a small-molecule inhibitor of Toll-like receptor (TLR) 4 signaling, binds selectively to TLR4 and interferes with interactions between TLR4 and its adaptor molecules. Mol Pharmacol 79: 34-41, 2011.

17. Visintin A, Halmen KA, Latz E, Monks BG and Golenbock DT: Pharmacological inhibition of endotoxin responses is achieved by targeting the TLR4 coreceptor, MD-2. J Immunol 175: 6465-6472, 2005.

18. Park EK, Jung HS, Yang HI, Yoo MC, Kim C and Kim KS: Optimized THP-1 differentiation is required for the detection of responses to weak stimuli. Inflamm Res 56: 45-50, 2007.

19. Fujita H, Yagishita N, Aratani S, Saito-Fujita T, Morota S, Yamano Y, Hansson MJ, Inazu M, Kokuba H, Sudo K, et al: The E3 ligase synoviolin controls body weight and mitochondrial biogenesis through negative regulation of PGC-1 $\beta$. EMBO J 34: 1042-1055, 2015

20. Walsh BW, Lenhart JS, Schroeder JW and Simmons LA: Far western blotting as a rapid and efficient method for detecting interactions between DNA replication and DNA repair proteins. Methods Mol Biol 922: 161-168, 2012.

21. Akashi S, Shimazu R, Ogata H, Nagai Y, Takeda K, Kimoto M and Miyake K: Cutting edge: Cell surface expression and lipopolysaccharide signaling via the toll-like receptor 4-MD-2 complex on mouse peritoneal macrophages. J Immunol 164 3471-3475, 2000

22. Horvatinovich JM, Grogan EW, Norris M, Steinkasserer A, Lemos H, Mellor AL, Tcherepanova IY, Nicolette CA and DeBenedette MA: Soluble CD83 inhibits T cell activation by binding to the TLR4/MD-2 complex on CD14 ${ }^{+}$monocytes. J Immunol 198: 2286-2301, 2017.
23. Krüger CL,Zeuner MT, Cottrell GS, Widera D and Heilemann M: Quantitative single-molecule imaging of TLR4 reveals ligand-specific receptor dimerization. Sci Signal 10: eaan1308, 2017.

24. Singh A, Singh V, Tiwari RL, Chandra T, Kumar A, Dikshit M and Barthwal MK: The IRAK-ERK-p67phox-Nox-2 axis mediates TLR4, 2-induced ROS production for IL-1 $\beta$ transcription and processing in monocytes. Cell Mol Immunol 13: 745-763, 2016.

25. Smith JA, Stallons LJ, Collier JB, Chavin KD and Schnellmann RG: Suppression of mitochondrial biogenesis through toll-like receptor 4-dependent mitogen-activated protein kinase kinase/extracellular signal-regulated kinase signaling in endotoxin-induced acute kidney injury. J Pharmacol Exp Ther 352: 346-357, 2015.

26. Luan H, Zhang Q, Wang L, Wang C, Zhang M, Xu X, Zhou H, $\mathrm{Li} \mathrm{X}, \mathrm{Xu} \mathrm{Q}, \mathrm{He} \mathrm{F}$, et al: OM85-BV induced the productions of IL-1 $\beta$, IL- 6 , and TNF- $\alpha$ via TLR4- and TLR2-mediated ERK1/2/NF- $\kappa \mathrm{B}$ pathway in RAW264.7 cells. J Interferon Cytokine Res 34: 526-536, 2014.

27. Cela I, Di Matteo A and Federici L: Nucleophosmin in its interaction with ligands. Int J Mol Sci 21: 4885, 2020.

28. Mitrea DM, Grace CR, Buljan M, Yun MK, Pytel NJ, Satumba J, Nourse A, Park CG, Madan Babu M, White SW, et al: Structural polymorphism in the N-terminal oligomerization domain of NPM1. Proc Natl Acad Sci USA 111: 4466-4471, 2014.

29. Chen X, Tao T, Wang H, Zhao H, Lu L and Wu F: Arterial thrombosis is accompanied by elevated mitogen-activated protein kinase (MAPK) and Cyclooxygenase-2 (COX-2) expression via Toll-like receptor 4 (TLR4) activation by S100A8/A9. Med Sci Monit 24: 7673-7681, 2018.

30. Watanabe T, Takahashi N, Hirabara S, Ishiguro N and Kojima T: Hyaluronan inhibits TLR4-dependent RANKL expression in human rheumatoid arthritis synovial fibroblasts. PLoS One 11: e0153142, 2016.

31. Blich M, Golan A, Arvatz G, Sebbag A, Shafat I, Sabo E, Cohen-Kaplan V, Petcherski S, Avniel-Polak S, Eitan A, et al: Macrophage activation by heparanase is mediated by TLR-2 and TLR-4 and associates with plaque progression. Arterioscler Thromb Vasc Biol 33: e56-e65, 2013.

32. Lu Z, Zhang X, Li Y, Lopes-Virella MF and Huang Y: TLR4 antagonist attenuates atherogenesis in LDL receptor-deficient mice with diet-induced type 2 diabetes. Immunobiology 220: 1246-1254, 2015

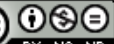

This work is licensed under a Creative Commons Attribution-NonCommercial-NoDerivatives 4.0 International (CC BY-NC-ND 4.0) License. 